\title{
Application of Off-Nadir Satellite Imagery in Earthquake Damage Assessment Using Object-Based HOG Feature Descriptor
}

\author{
S. Jabari ${ }^{1, *}$, M. Krafczek ${ }^{1}$ \\ ${ }^{1}$ Department of Geodesy and Geomatics Engineering, Faculty of Engineering, Fredericton, NB, Canada
}

(sh.jabari, Mitchell.Krafczek)@unb.ca

KEY WORDS: off-nadir satellite imagery, damage detection, Object-Based Image Analysis, HOG feature descriptor

\begin{abstract}
:
One of the most crutial applications of very-high-resolution (VHR) satellite images is disaster management. In disaster management, time is of great importance. Therefore, it is vital to acquire satellite images as quickly as possible and benefit from automatic change detection to speed up the process. Automatic damage map generation is performed by overlaying the co-registered before and after images of the area of interest and, compring them to highlight the affected infrastructures. For speeding up image capture, satellites tilt their imaging sensor and take images from oblique angles. However, this kind of image acquisition causes severe geometric distortion in the images, which hinders image co-registration in automatic change detection. In this study, a Patch-Wise Co-Registration (PWCR) solution is used. In this algorithm, the before and after images are co-registered in a segment-by-segment manner. From the literature, this algorithm is followed by a spectral comparison to detect changes. However, due to the complicated structure of debris in damage detection applications, spectral comparison methods cannot perform well. In this work, we developed an object-based method using Histogram of Oriented Gradient descriptor to detect damges and compared our results to different existing spectral and textural change detection methods. The algorithm is tested on images from the 2010-Heidi earthquake, captured by DigitalGlobe. The achieved highly accurate results demonstrate the potential of using off-nadir remote sensing images for automatic urban damage detection possibly in early response systems as it speeds up the damage map generation by providing flexibility to utilize images taken from different anlges.
\end{abstract}

\section{INTRODUCTION}

Structural damage assessment is essential for post-disaster relief efforts. Remote sensing-based damage detection is amongst the most popular methods of damage map generation. Using remote sensing, a detailed spatial information of a disturbed area can be generated which can provide almost real-time evidence of building conditions using a variety of sensors such as optical, SAR (synthetic aperture radar) and LiDAR (light detection and ranging) (Khoshelham et al., 2013; Uprety, Yamazaki, 2012). In literature, there are numerous methods used for disaster change detection (Donga, Shan, 2013). Khoshelham et al., 2013 used aerial laser scanning data and segment-based classification of damaged buildings to assess damage to local buildings. Vetrivel et al., 2016 present a method for automatic classification and segmentation of satellite images. Uprety and Yamazaki, 2012 assessed building damage using high-resolution SAR imagery while (Homa Zakeri, Wen Liu, Fumio Yamazaki, Takashi Nonaka, 2016) used both SAR and optical data. Both groups stated that without the proper correlation coefficients and backscatter values from the pre- and post-event intensity images, they were unable to achieve optimal results. It was concluded that more reliable ground truth data was required to detect building damage. In the literature, there are other methods of damage detection which are based on using convolution neural networks (CNN) or deep learning to assess building damages (Girshick et al., 2012). The majority of the studies in the literature are based on using nadir images or existing 3D models which are not always available in the case of a disaster. The nature of disaster management requires methods that are flexible enough to benefit from the quickly captured images. The quickly captured images are usually the ones that are taken with an off-nadir angle. The agility technology of the satellites allows them to tilt their sensors and acquire images from an off-nadir angle. However, since the majority of change detection methods are based on a perfect pixel-to-pixel registration between the before and after images, off-nadir images cannot be effectively used in automatic change detection methods. Jabari, Zhang, 2016 proposed a method of patch-wise co-registration (PWCR) between the before and after images that are taken from off-nadir angles. The PWCR method has reported being useful for the image-to-image registration and detection of changes using spectral comparison methods.

Spectral comparison of the before and after images in the damage detection is not recommended as the spectral signature of the impervious surfaces does not drastically change when damaged (Li et al., 2011). However, the texture of the features changes due to the chaotic distribution of the debris of collapsed built-up structures after the damages (Pesaresi et al., 2007). Thus, in the literature, textural parameters are reported to be successful in damage detection (Bignami et al., 2011; Pesaresi et al., 2007; Tomowski et al., 2010).

Recently, the Histogram of Oriented Gradient (HOG) descriptor is being increasingly used for different computer vision applications (Bertozzi et al., 2007; Memarzadeh et al., 2013). The fact that HOG is sensitive to local variations in the brightness values makes it suitable for damage detection as it has been used in (Vetrivel et al., 2016). Vetrivel et al. 2016 used a HOG feature descriptor and a Gabor wavelet function along with SVM and Random Forest classifiers to develop a damage-based classification. These, along with a bag of words, allowed them to identify and extract damaged areas. The HOG feature descriptor uses a fixed window around points of interest to detect the dominant direction of gradients.

Considering the nature of off-nadir images, the direction of relief displacement in elevated objects varies depending on the viewing angle of the images. Therefore, the geometric properties of the bi-temporal off-nadir images can be different. Thus, a fixed window solution is not suitable for change detection. To the best of our knowledge there is no study in the literature that uses a

* Corresponding author 
flexibly-shaped neighbourhood for HOG feature descriptor generation. The hypothesis of this study is whether an objectbased approach for generating the HOG feature descriptor using off-nadir satellite images can distinguish damages from nondamages.

The main contribution of this study is to combine PWCR image registration along with an object-based (OB) approach as the effective neighbourhood of Histogram of Oriented Gradient (HOG) descriptor to find changes between before and after disaster images.

\section{METHODOLOGY}

The methodology of the proposed process is presented in Figure 1.

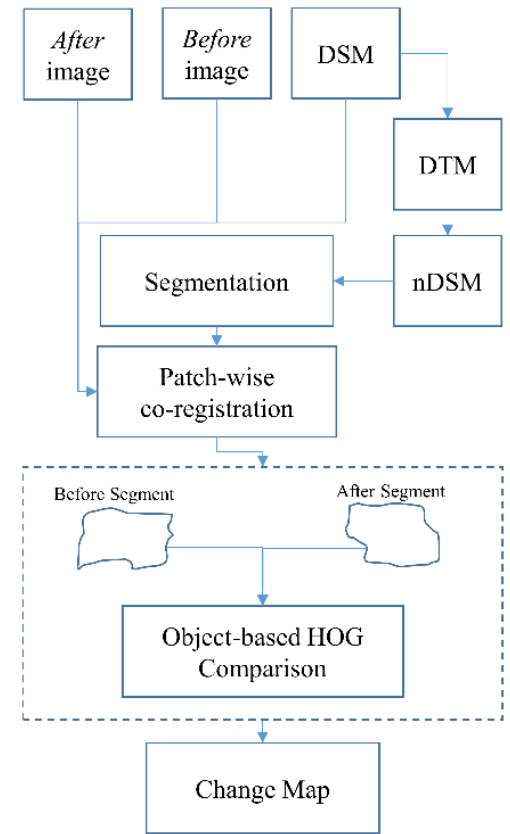

Figure 1: Proposed method of damage detection

As in every change detection method, the first step of this work is the co-registration. Considering the different view-angle of the images acquired at different times, it is not possible to use traditional co-registration methods such as the ones that are based on ortho-rectification and then co-registration using standard polynomial-based methods. Thus, a co-registration method is required, which is robust against view angle differences of the images. This solution is proposed in (Jabari, Zhang, 2016). Section 2.1 contains more details about this method of image registration. After finding the corresponding segments in the before and after images, a comparison method is required to identify damages from non-damages. In this study, HOG feature descriptor is used. Section 2.2 presented details about this comparison method.

\subsection{Patch-wise co-registration}

As reported in (Jabari, Shabnam; Zhang, 2018), to overcome the problem of different view-angles of the remote sensing images, the base image can be divided into segments. Then, using the Rational Function Model projection, along with a Digital Surface Model (DSM) of the area, the segments can be projected to the other image (target image). Therefore, the registration is performed in a segment-by-segment manner. If the building footprint map of the area is available, using Rational Polynomial Coefficients (RPCs), the building polygons can be projected from the object space (DSM space) into the images. The model with which the projection is performed is called the Rational Function Model (RFM) (Tao, Hu, 2001).

However, if the building footprints are not available, it is still possible to detect buildings if the DSM has a high spatial resolution such as the DSMs generated from high-density LiDAR data. Figure 2 shows how the building segments are generated from a high-spatial-resolution DSM and how the building segments are projected to the image spaces which accordingly results in co-registration between the base image and the target image in a segment-wise manner.

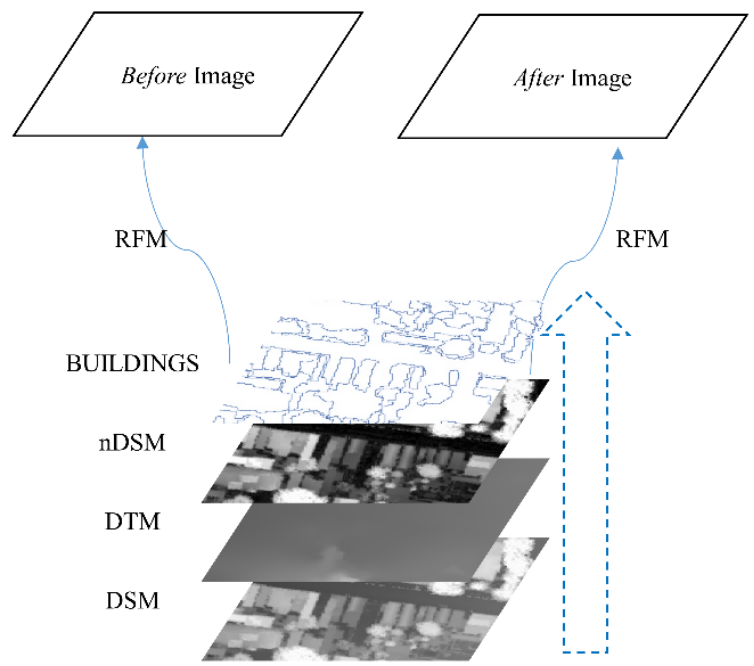

Figure 2: Extracting building segments and performing PWCR.

As shown in Figure 2, first, a Digital Terrian Model (DTM) is generated by filtering the DSM; second, nDSM=DSM-DTM is attained; finally, the nDSM is segmented to ideanity elevated object bodeders. To remove noise, only objects with high rectangular-fit value are used as candidate buildings. To differenciate buildings from trees, all the candidate building segments are projected to the image spaces and the ones with high Normalized Difference Vegetation Index (NDVI) values are removed from the segments of interest. It is worth noting that further segment adjustment is possible to achieve more smooth building borders which is out of the scope of this study.

\subsection{Object-based HOG feature descriptor}

To generate HOG feature descriptor for a given image, $f(x, y)$, first the intensity of gradient, $|\nabla f(x, y)|$, and the orientation of the intensity gradient, $\theta(x, y)$, of the image are generated. Then, within a given window of $m \times n$, the directions are binned into $t$ bins, each bin covering $\pi / \mathrm{t}$ radians, and each pixel casts a weighted vote for a bin depending on the magnitude of the gradient of that pixel. Then, the histogram values are normalized within a block of windows (Memarzadeh et al., 2013).

Due to the off-nadir angle of the images used in this study, it is not possible to use fixed windows, of size $m \times n$, for HOG feature descriptor. Thus, we developed an object-based version of the HOG feature descriptor, which takes into account the shape of the objects instead of using a fixed window throughout the images.

In this OB-HOG method, the gradient and direction of all the pixels in a segment are used to generate the HOG feature descriptor of that segment.

As shown in Figure 3, the same process is followed for the corresponding segments in bi-temporal. Then, the difference between the HOG feature descriptors of corresponding segments is considered as a criterion for change. Respecting the nature of 
the HOG feature descriptor, which reflects the dominant edges in the area, more dominant directions is expected in the nondamaged areas while more chaotic HOG distribution is anticipated in damaged ones.

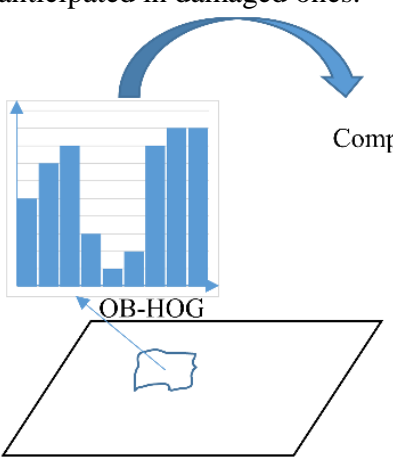

Before Image

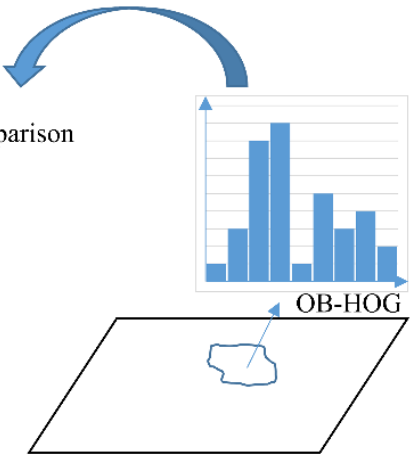

After Image
Figure 3: The process of using Object-Based HOG feature descriptor for damage detection. The HOG feature descriptor is generated over corresponding segments and then compared in order to detect changes.

\section{DATASETS}

To study the effect of using PWCR along with OB-HOG feature descriptor, we used the Haiti 2010 earthquake as a case study. The magnitude-7.0 earthquake shocked Haiti on January $12^{\text {th }}$, 2010, destroying several buildings in the country.

We used two WorldView-2 (WV2) images of the area acquired before and after the shock. Table 1 shows details about the data used.

Table 1:Metadata of the data used

\begin{tabular}{|l|l|l|l|}
\hline Data & Before & \multicolumn{1}{|c|}{ After } & LiDAR data \\
\hline Acquisition date & $1 / 9 / 2010$ & $1 / 15 / 2010$ & $01 / 21 / 2010-$ \\
& & & $01 / 27 / 2010$ \\
GSD(m) /LiDAR & 0.551 & 0.523 & 3.4 \\
$\begin{array}{l}\text { point density } \\
\text { (pts/m2) }\end{array}$ & & & \\
Off-nadir angle $\left(^{\circ}\right)$ & 25.1 & 19.1 & -- \\
Sensor azimuth $\left({ }^{\circ}\right)$ & 321.3 & 61.4 & 315 \\
Sensor elevation $\left({ }^{\circ}\right)$ & 61.8 & 68.6 & 45 \\
\hline
\end{tabular}

The LiDAR data was further processed to generate a DSM, and accordingly DTM and nDSM, of the area with the Ground Sampling Distance (GSD) of $0.5 \mathrm{~m}$, which is similar to the GSDs of the satellite images.

Figure 4 shows snapshots of the images/DSM used in this study.

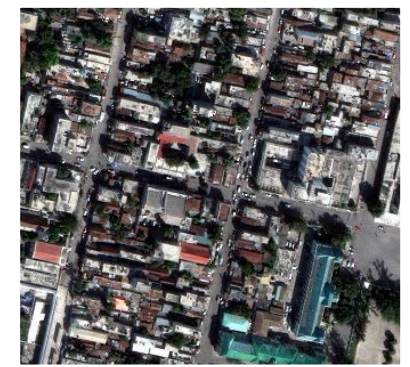

(a)

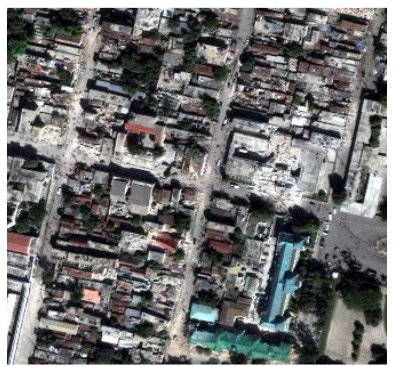

(b)

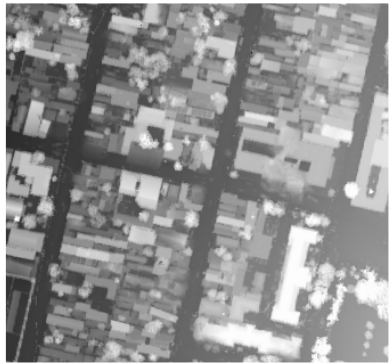

(c)

Figure 4: snapshots of (a) before, (b) after, (c) DSM of the area used in this study.

\section{RESULTS AND DISCUSSION}

As mentioned in section 2.2, since a building footprint layer representing all the buildings was not available to us, we used the DSM of the area to detect buildings first. Then, the images are co-registered using PWCR. Please see (Jabari, Zhang, 2016) for more details.

Due to the inherent errors in the RPCs of the satellite images, a final adjustment is required to remove the RPC biases. Thus, a polynomial degree-one registration is used between the projected footprints and the original locations of the points of interest. Finally, the OB-HOG difference of the corresponding segments in the before and after images was used as a change criterion. To compare the accuracy of the proposed OB-HOG method with other methods used for damage detection, two groups of change detection methods are used. Group (1) spectral comparison-based change detection methods that include: Change Vector Analysis (CVA), Correlation Analysis, Cosine Angle, and Multi-variate Alteration Detection (MAD); Group (2) texture comparisonbased change detection methods that include: Contrast, Dissimilarity, Entropy, and Homogeneity. For more details on the comparison methods refer to (A., Jia, 1999; Haralick, 1979; Nielsen, 2011).

We selected a group of changed and a group of unchanged buildings, totalling around 100 buildings as our test case in this study.

To test the performance of the proposed OB-HOG method against other spectral/textural methods, we used the Receiver Operating Characteristic curves that plot True Positive Rate (TPR) versus False Positive Rate (FPR). Equation 1 presents the formula for TPR and FPR.

$$
\begin{aligned}
& T P R=\frac{t p}{t p+f n} \\
& F P R=\frac{f p}{f p+t n}
\end{aligned}
$$

where

$$
\begin{aligned}
& \mathrm{tp}=\text { true positive } \\
& \mathrm{fn}=\text { false negative } \\
& \mathrm{fp}=\text { false positive }
\end{aligned}
$$$$
\mathrm{tn}=\text { true negative }
$$

Figure 5 shows the the ROC curves of the OB-HOG method along with the spectral and textural comparison methods. The closer the ROC curve to the point $(01$,$) the higher the$ performance of the parameter in distinguishing two classes (here changed and unchanged classes). 


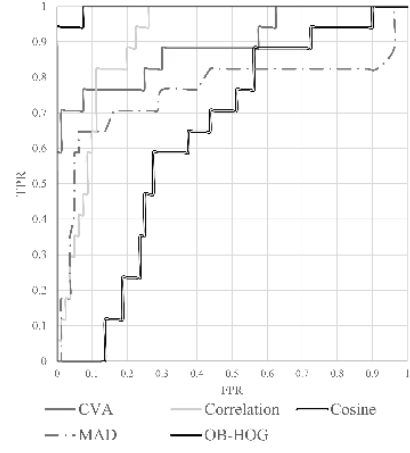

(a)

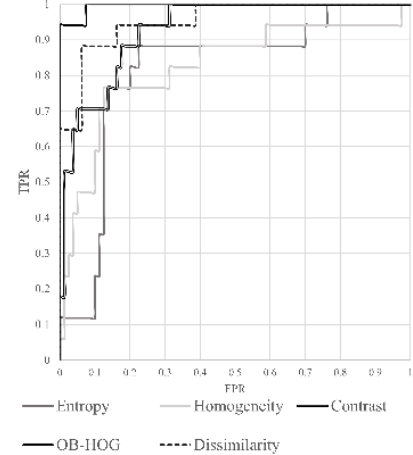

(b)
Figure 5: ROC curves of the OB-HOG and the (a) spectral (b) textural change detection methods.

Another parameter which can quantitatively compare the ROC curves is called Area Under Curve, which integrates the area under the ROC curves. The higher the AUC value of the change detection criterion, the better the parameter in differentiating two classes (here changes from non-changes). Figure 6 shows the the ROC curves of the OB-HOG method along with the spectral and textural comparison methods.

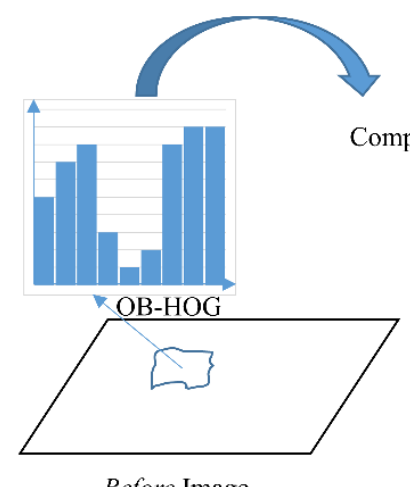

(a)

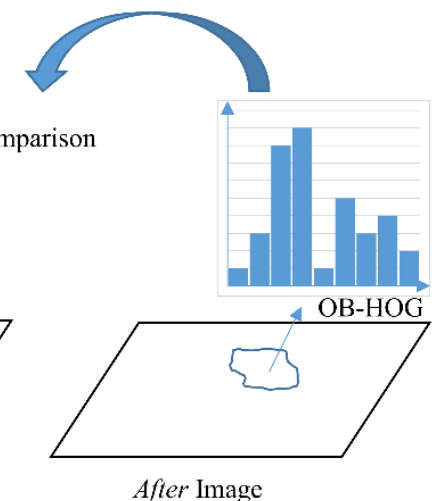

(b)
Figure 6: AUC of the OB-HOG with the ROC curve of the (a) spectral (b) textural change detection methods.

As can be seen in Figures 5 and 6, OB-HOG over-performs all other comparison methods checked in this study.

Furthermore, as expected, textural methods perform better than spectral comparison methods. The reason is that by the destruction caused by disasters, like an earthquake, generally, the material of the objects does not change. Therefore, spectral comparison methods can underperform in finding changed objects. However, textural parameters consider the local distribution of the pixel values, which can better highlight changes due to existence of debris. The HOG feature descriptor is also based on the gradient image, which is of the same nature as textural parameters. However, the fact that HOG feature descriptor incorporates the gradient value along with the direction of the gradient value makes it superior compared to other texture-based change detection methods.

By combining PWCR co-registration along with the object-based HOG feature descriptor, a good differenciation between damaged and non-damaged areas have been achieved. This process needs to be followed by a proper threshold selection method to classify damaged buldings from non-damaged ones in a future study. It $\mathrm{s}$ worth noting that this method performs well in typical flatroofed buildings as they usually do not have dominant edges inside the building border. However, the buildings with variant architectural shapes can generate shadows in different directions depending on the solar azimuth and elevation angles, which can cause false-alarm change detection results.

Another challenge in this study was that the LiDAR data, was acquired after the disaster. Thus, the process of detecting buildings from the DSM was not $100 \%$ successful. We missed a few totally collapsed buldings as their nDSM values were not high enough to be detected as buildings. For future practice, it is recommended to use the LiDAR data of before disaster or use building footprints - both of which was not available to us for the study area.

Besides, in this case, only the roofs of the buildings are analysed for damage detection. Due to the eagle-eye looking angle of the satellite images, it is not possible to detect damages to the building façade. This issue can be addressed using oblique airborne or UAV images in a future study.

\section{CONCLUSION}

In this paper, we investigated using off-nadir images for damage detection after a natural disaster like an earthquake. Off-nadir images form the majority of the VHR satellite imagery archives produced by imagery vendors. Besides, the quickest images acquired after an inevitable disaster can have an off-nadir angle due to using the agility technology of satellites to tilt their sensors and acquire images from areas further away from their footprints on the ground. Thus, automatic damage detection methods using off-nadir imagery are essential.

In this paper, we used a patch-wise co-registration method to find the corresponding buildings in the off-nadir before and after images. Then, we developed an object-based version of HOG feature descriptor to looks for damaged buildings by comparing the before and after images of the area of interest. We compared our results to other change detection methods based on spectral and textural comparisons, and our proposed method outperformed them. This method has the potential to be used over the quickest images taken from a disturbed area and compare them to existing imagery from the archives to detect damages in the area.

\section{ACKNOWLEDGEMENTS}

This research has been supported by University of New Brunswick URF and Natural Sciences and Engineering Research Council (NSERC) Canada. The worldview 2 images used in this study are kindly provided by DigitalGlobe Foundation. We would like to thank Rochester Institute of Technology and Kucera International (under sub-contract to ImageCat Inc. and funded by the Global Facility for Disaster Reduction and Recovery (GFDRR) hosted at The World Bank) for providing the LiDAR data. This work is based on the LiDAR data provided by the OpenTopography Facility with support from the National Science Foundation under NSF Award Numbers 1557484, 1557319, and 1557330 (DOI: 10.5069/G96Q1V50).

\section{REFERENCES}

A., J. R., Jia, X. (Eds.). 1999. Remote Sensing Digital Image Analysis (3rd ed.). Springer.

Bertozzi, M., Broggi, A., Del Rose, M., Felisa, M., Rakotomamonjy, A., Suard, F. 2007. A pedestrian detector using histograms of oriented gradients and a support vector machine classifier. 2007 IEEE Intelligent Transportation Systems Conference, 143-148. 
Bignami, C., Chini, M., Stramondo, S., Emery, W. J., Pierdicca, N. 2011. Objects textural features sensitivity for earthquake damage mapping. 2011 Joint Urban Remote Sensing Event, JURSE $2011 \quad$ - $\quad$ Proceedings, 333-336. https://doi.org/10.1109/JURSE.2011.5764787

Donga, L., Shan, J. 2013. A comprehensive review of earthquake induced building damage detection with remote sensing techniques. International Society for Photogrammetry and Remote Sensing, Inc. (ISPRS), 84, 85-99.

Girshick, R., Donahue, J., Darrell, T., Berkeley, U. C., Malik, J. 2012. (r-cnn) Rich feature hierarchies for accurate object detection and semantic segmentation. 2014 IEEE Conference on Computer Vision and Pattern Recognition, 2-9. https://doi.org/10.1109/CVPR.2014.81

Haralick, R. M. 1979. Statisti1. Haralick, R. M. Statistical and structural approaches to texture. Proc. IEEE 67, 786-804 (1979).cal and structural approaches to texture. Proceedings of the IEEE, 67(5), 786-804.

Homa Zakeri, Wen Liu, Fumio Yamazaki, Takashi Nonaka, T. S. 2016. Building Damage Assessment in the 2010 Haiti Earthquake Using High Resolution Building Damage Assessment in the 2010 Haiti. (October), 2-5.

Jabari, Shabnam; Zhang, Y. 2018. Urban Change Detection Utilizing High-Resolution Optical Images Taken from Different Viewing Angles and Different Platforms. In P. Weng, Qihao; Quattrochi, Dale; Gamba (Ed.), Urban Remote Sensing (2nd ed., pp. (125-148). https://doi.org/10.1201/9781138586642

Jabari, S., Zhang, Y. 2016. RPC-Based Coregistration of VHR Imagery for Urban Change Detection. Photogrammetric Engineering, Remote Sensing, 82(7), 521-534. https://doi.org/10.14358/PERS.82.7.521

Khoshelham, K., Oude Elberink, S., Xu, S. 2013. Segment-based classification of damaged building roofs in aerial laser scanning data. IEEE Geoscience and Remote Sensing Letters, 10(5), 12581262. https://doi.org/10.1109/LGRS.2013.2257676

Li, P., Xu, H., Song, B. 2011. A Novel Method for Urban Road Damage Detection Using Very High Resolution Satellite Imagery and Road Map. 1057-1066.

Memarzadeh, M., Golparvar-Fard, M., Niebles, J. C. 2013. Automated 2D detection of construction equipment and workers from site video streams using histograms of oriented gradients and colors. Automation in Construction, 32, 24-37. https://doi.org/10.1016/j.autcon.2012.12.002

Nielsen, A. A. 2011. Kernel maximum autocorrelation factor and minimum noise fraction transformations. Image Processing, IEEE Transactions On, 20(3), 612-624.

Pesaresi, M., Gerhardinger, A., Haag, F. 2007. Rapid damage assessment of built-up structures using VHR satellite data in tsunami-affected areas. International Journal of Remote Sensing, 28(13-14), https://doi.org/10.1080/01431160601094492

Tao, C. V, Hu, Y. 2001. A comprehensive study of the rational function model for photogrammetric processing. Photogrammetric Engineering and Remote Sensing, 67, 13471357.
Tomowski, R., Klonus, S., Ehlers, M., Michel, U., Reinartz, P. 2010. Change Visualization Through a Texture-Based Analysis Approach for Disaster Applications. ISPRS TC VII Symposium 100 Years ISPRS, XXXVIII(May 2014), 263-268.

Uprety, P., Yamazaki, F. 2012. Detection of Building Damage in the 2010 Haiti Earthquake Using High-Resolution Sar Intensity Images. Journal of Japan Association for Earthquake Engineering, 12(6), 6_21-6_35. https://doi.org/10.5610/jaee.12.6_21

Vetrivel, A., Kerle, N., Gerke, M., Nex, F., Vosselman, G. 2016. Towards automated satellite image segmentation and classification for assessing disaster damage using data-specific features with incremental learning. https://doi.org/10.3990/2.369

Vetrivel, Anand, Gerke, M., Kerle, N., Vosselman, G. 2016. Identification of structurally damaged areas in airborne oblique images using a Visual-Bag-of-Words approach. Remote Sensing, 8(3). https://doi.org/10.3390/rs8030231 